\title{
The association between Hepcidin and arterial stiffness in a community-dwelling population
}

\author{
Xiaona Wang ${ }^{\dagger}$, Li Sheng ${ }^{\dagger}$, Ping Ye*, Ruihua Cao, Xu Yang, Wenkai Xiao, Yun Zhang, Yongyi Bai and Hongmei Wu
}

\begin{abstract}
Background: An association of hepcidin with cardiovascular (CV) disease and atherosclerosis has been reported in different patient groups. However, it has not been well described clinically the association between hepcidin and arterial stiffness. In this study,We analysed the possible mechanism of Hepcidin and arterial stiffness.

Methods: This article related measurements of plasma hepcidin and arterial stiffness (carotid-femoral pulse wave velocity [PWV]) in a community-based sample.

Results: After a median follow-up interval of 4.8 years, multiple linear regression analysis revealed that hepcidin was independently associated with carotid-femoral PWV $(\beta=1.498, P<0.001)$. In a multivariable linear regression analysis, HDL3-C levels were negatively and independently associated with hepcidin at baseline ( $\beta=-0.857, P=0.024)$. HDL2-C was not associated with hepcidin at baseline $(\beta=-1.121, P=0.133)$.

Conclusions: We found an association between baseline hepcidin and follow-up arterial stiffness that was independent of age, gender and other vascular risk factors. We also identified an association between hepcidin and HDL3-C at baseline, which indicates that the HDL3-C level may reflect the change in cholesterol efflux from peripheral arteries and partly explain the relationship between hepcidin and the change of arterial stiffness.
\end{abstract}

Keywords: Hepcidin, HDL3-C, Carotid-femoral pulse wave velocity

\section{Background}

An abnormality in iron metabolism has been recognized as a strong predictor of cardiovascular disease [1, 2]. The Kuopio Ischaemic Heart Disease Risk Factor Study including 1931 Finnish men with asymptomatic for heart disease confirmed that elevated stored iron levels increase the incidence of coronary heart disease [3]. Meanwhile, the Bruneck Research found that the incidence of atherogenesis in male patients is three times that of premenopausal women which demonstrates an a strong correlation between high levels iron and atherogenesis [4]. Hepcidin was first discovered as a peptide with antimicrobial properties in 2001 [5, 6]. Recently, hepcidin has been recognized as the key hormone in the regulation of iron balance and iron recycling $[7,8]$. Some studies have reported a

\footnotetext{
*Correspondence: yeping301@sina.com

${ }^{\dagger}$ Xiaona Wang and Li Sheng contributed equally to this work.

Department of Geriatric Cardiology, Chinese PLA General Hospital, Fuxing

Road \#28, Beijing 100853, China
}

strong association between hepcidin and cardiovascular (CV) disease and atherosclerosis in different patient groups [9-11]. However, mechanistic studies have mainly focused on hepcidin-mediated iron metabolism.

Recently, the pharmacologic suppression of hepcidin has been shown to increase the expression of ABCA1 and ABCG1 and lipid efflux via the macrophage-specific expression of cholesterol efflux transporters and to reduce foam cell formation and atherosclerosis in vivo and vitro [12]. Reverse cholesterol transport (RCT) is the main process of HDL that opposes atherosclerosis. Great emphasis has been placed on the role of individual HDL subclasses (HDL2-C, HDL3-C), which are not equally atheroprotective [13]. Therefore, the attention on HDL-C has now started to transfer away from the cholesterol-centric theory towards alternative indices of HDL such as subclass distribution, particle size, and measures of HDL functionality. However, what is lacking is a clinical study on hepcidin and HDL subclasses. 
Carotid-femoral PWV has been generally regarded as a "gold standard" indicator of subclinical vascular disease as well as cardiovascular mortality in many epidemiological studies [14]. In this article, we puts forward the relationship between hepcidin and arterial stiffness by studying: (1) the predictive connection of baseline hepcidin values with follow-up arterial stiffness in a large longitudinal sample in Beijing; and (2) the relationship between HDL subclasses with hepcidin at baseline to explain a possible clinical mechanism.

\section{Methods}

\section{Subjects}

The population in this study included 1447 subjects who lived in Shijingshan community Pingguoyuan area of Beijing, China. The original data was from a routine health check-up of 1680 subjects between September 2007 and January 2009. We conducted the first follow-up evaluation to collect information from these subjects prospectively from February 1 to September 30, 2013, and 1499 subjects were successfully evaluated (181 subjects were lost, follow-up rate was $89.2 \%$ ). The median follow-up interval for the original 1499 subjects was 4.8 years. The final number of subjects is $1447 ; 52$ samples were excluded (death). No differences other than in baseline risk factors were noted in participants who completed baseline and follow-up assessments.

\section{Clinical data collection}

Clinical data were collected via a face-to-face questionnaire survey from all subjects to ascertain new CVD events during these visits. Data were categorized as below:

- Data about prevalent diseases, medical histories, lifestyle factors and family history;

- Data about urine and fasting blood samples;

- Data about blood pressure and other anthropometric measurements (obtained by trained physicians).

\section{Biomarker variable determination}

Blood samples were collected in centrifuge tubes after subjects fasted overnight and were centrifuged them for $15 \mathrm{~min}$ at $1200 \times \mathrm{g}$. Serum aliquots were placed at $-80{ }^{\circ} \mathrm{C}$ before use. Hepcidin concentrations were measured using a commercially available quantitative sandwich ELISA assay (CY-8079; CycLex Co., Nagano, Japan). HDL 2 and HDL 3 were separated in a Himac centrifuge with a PR80A rotor (Hitachi, Tokyo, Japan) [15]. Concentrations of fasting blood glucose (FBG), high-density lipoprotein cholesterol (HDL-C), low-density cholesterol cholesterol (LDL-C), triglyceride (TG) and total cholesterol (TC) were evaluated on a Roche autoanalyzer (Roche Diagnostics, Indianapolis, IN, USA). Concentrations of serum creatinine were measured on Hitachi 7600 autoanalyser (Hitachi, Tokyo, Japan). All testing was performed in the same laboratory following the criteria of the World Health Organization Lipid Reference Laboratories.

\section{Assessment of arterial stiffness}

Before the experiment, everyone avoided smoking, drinking, tea and caffeine. Arterial stiffness was measured and calculated by carotid-femoral pulse wave velocity (cf-PWV) using Complior SP device (Createch Industrie, Massy, France). We positioned two pressure-sensitive transducers (Fukuda Denshi Co., Tokyo, Japan) over the common carotid artery and the femoral artery. Arterial stiffness was calculated according to the following formula: cf-PWV $(\mathrm{m} / \mathrm{s})=$ distance $(\mathrm{m}) /$ transit time $(\mathrm{s})$ [16].

\section{Definition of variables}

- Body mass index $(\mathrm{BMI})=$ weight $(\mathrm{kg}) /$ height $^{2}\left(\mathrm{~m}^{2}\right)$.

- The estimated glomerular filtration rate (eGFR) was calculated via the below Chronic Kidney Disease Epidemiology Collaboration equation: eGFR $=141 \times$ $\min (\mathrm{Scr} / \kappa, 1) \alpha \times \max (\mathrm{Scr} / \kappa, 1)-1.209 \times 0.993 \mathrm{Age} \times$ 1.018 [if female] $\times 1.159$ [if black], where Scr is plasma creatinine $(\mathrm{mg} / \mathrm{dL}), \mathrm{K}$ is 0.7 for females and 0.9 for males, $\alpha$ is -0.329 for females and -0.411 for males, min indicates the minimum of $\mathrm{Scr} / \mathrm{K}$ or 1 , and max indicates the maximum of $\mathrm{Scr} / \mathrm{k}$ or 1 .

\section{Statistical analyses}

Median (interquartile range) or mean \pm standard deviation (SD) was used to expressed baseline continuous variables were expressed as the. Percentages were used to expressed dichotomous variables.

Two categories were classified according to baseline distribution of Carotid-femoral PWV:

1) elevated levels $(\geq 12 \mathrm{~m} / \mathrm{s})$;

2) normal level $(<12 \mathrm{~m} / \mathrm{s})$ [17].

We used t-test to describe baseline continuous variables and chi-square to describe baseline categorical variables between elevated and normal carotid-femoral PWV groups.

Pearson's correlation and multiple linear regression analysis were used to describe the correlations between the baseline hepcidin level and follow-up carotid-femoral PWV. Regression models were adjusted for age and gender as well as hypertension, SBP, DBP, Diabetes (DM), smoking, BMI, levels of glucose, TC, HDL-C, TG, LDL-C and eGFR. In addition, we investigated the association of hepcidin in combination with HDL subclasses (HDL2-C, HDL3-C) at baseline by leveraging Pearson's correlation and multiple linear regression analysis. 
We used receiver operating characteristic (ROC) curves to assess the ability of the baseline hepcidin level indices to predict carotid-femoral PWV.

We used SPSS software for Windows, version 13.0 (SPSS, Chicago, IL, USA). $P$-values of $<0.05$ were considered statistically significant.

\section{Results}

\section{Baseline parameters in elevated and normal carotid-} femoral PWV groups

Characteristics of all 1447 subjects without cardiovascular disease at baseline according to baseline carotid-femoral PWV are summarized in Table 1 . The mean age $( \pm$ SD) of the population in the study was $61.30 \pm 11.4$ years. Age, levels of SBP, FBG, TG, LDL-C and hepcidin, as well as rates of male gender and smoking were significantly higher, but eGFR and HDL3-C levels were lower in the elevated carotid-femoral PWV group compared with normal cfPWV group.

\section{Association of baseline hepcidin with follow-up carotid-femoral PWV}

Age $(r=0.538 ; P<0.001)$, hepcidin $(r=0.151 ; P<0.001$, Fig. 1), TG $(r=0.747 ; P<0.001)$, SBP $(r=0.228 ; P<$ $0.001)$ and FBG $(r=0.118 ; P<0.001)$ were related to carotid-femoral PWV significantly and positively. eGFR $(r=-0.376 ; \mathrm{P}<0.001)$ has a inversely relationship with carotid-femoral PWV.

Table 2 showed the relationship between baseline hepcidin and follow-up arterial stiffness in multivariable

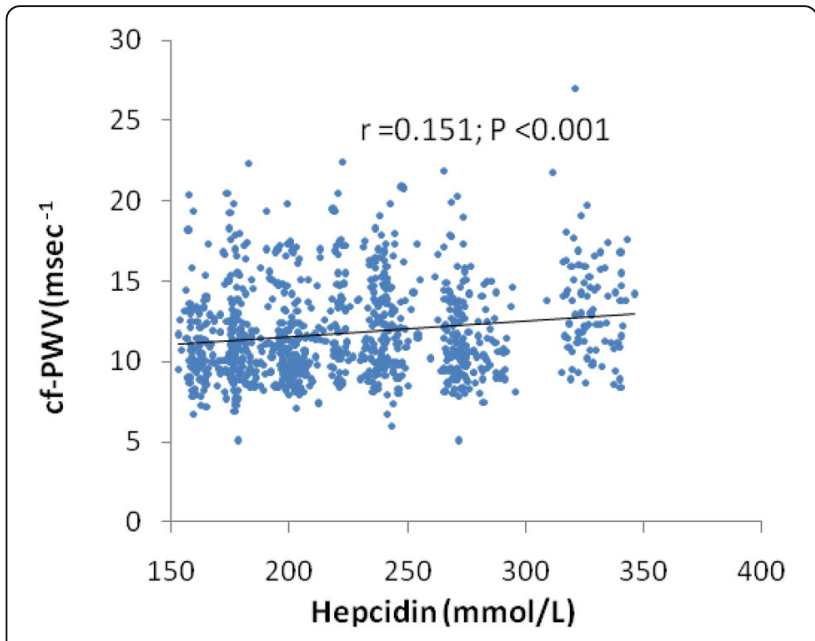

Fig. 1 Association of baseline hepcidin with follow-up Carotid-femoral PWV

linear regression analysis. Follow-up carotid-femoral PWV had a positively and independently association with baseline hepcidin level $(\beta=1.498, \mathrm{P}<0.001)$. Furthermore, male gender, hypertension, TG, LDL-C, TC, SBP, BMI and fasting blood glucose levels were positively and independently associated with carotid-femoral PWV whereas eGFR was negatively associated with follow-up carotid-femoral PWV.

ROC curves for the assessment of the hepcidin level indices as predictors of carotid-femoral PWV are presented in Fig. 2.

Table 1 Baseline characteristics of the subjects

\begin{tabular}{|c|c|c|c|c|}
\hline Variables & $\begin{array}{l}\text { All subjects } \\
(n=1447)\end{array}$ & $\begin{array}{l}\text { Carotid-femoral PWV } \geq 12 \\
(n=571)\end{array}$ & $\begin{array}{l}\text { Carotid-femoral PWV }<12 \\
(n=876)\end{array}$ & $P$-value \\
\hline Age & $61.30 \pm 11.4$ & $65.59 \pm 9.03$ & $54.14 \pm 10.11$ & $<0.001$ \\
\hline Male (\%) & $601(41.53)$ & $270(47.28)$ & 331 (37.78) & $<0.001$ \\
\hline $\mathrm{BMI}$ & $25.41 \pm 3.32$ & $25.41 \pm 3.28$ & $25.26 \pm 4.17$ & 0.583 \\
\hline $\mathrm{SBP}(\mathrm{mmHg})$ & $128.7 \pm 17.7$ & $135.38 \pm 18.37$ & $124.37 \pm 15.93$ & $<0.001$ \\
\hline $\mathrm{DBP}(\mathrm{mmHg})$ & $77.11 \pm 10.26$ & $77.00 \pm 10.92$ & $77.18 \pm 9.82$ & 0.762 \\
\hline FBG (mmol/l) & $5.39 \pm 1.65$ & $5.63 \pm 1.87$ & $5.21 \pm 1.35$ & $<0.001$ \\
\hline $\mathrm{TG}(\mathrm{mmol} / \mathrm{l})$ & $1.80 \pm 1.24$ & $1.92 \pm 1.27$ & $1.74 \pm 1.24$ & 0.017 \\
\hline $\mathrm{TC}(\mathrm{mmol} / \mathrm{l})$ & $5.01 \pm 0.93$ & $5.02 \pm 0.97$ & $5.01 \pm 0.89$ & 0.845 \\
\hline $\mathrm{HDL}-\mathrm{C}(\mathrm{mmol} / \mathrm{l})$ & $50.83 \pm 13.02$ & $50.32 \pm 12.60$ & $51.47 \pm 13.33$ & 0.191 \\
\hline HDL2-C & $29.85 \pm 9.13$ & $29.69 \pm 9.44$ & $30.05 \pm 9.08$ & 0.593 \\
\hline HDL3-C & $20.98 \pm 4.22$ & $20.62 \pm 4.12$ & $21.42 \pm 4.38$ & 0.006 \\
\hline LDL-C(mmol/l) & $2.91 \pm 0.71$ & $2.96 \pm 0.73$ & $2.87 \pm 0.69$ & 0.030 \\
\hline eGFR (ml/min) & $94.2 \pm 14.30$ & $88.18 \pm 14.19$ & $98.63 \pm 12.45$ & $<0.001$ \\
\hline Hepcidin & $226.43 \pm 45.53$ & $209.94 \pm 43.34$ & $227.20 \pm 48.83$ & $<0.001$ \\
\hline Smokers & $380(26.26)$ & 175 (30.65) & $205(23.40)$ & $<0.001$ \\
\hline Hypertension & $755(52.17)$ & $418(73.20)$ & $337(38.47)$ & $<0.001$ \\
\hline
\end{tabular}

$B M I$ body mass index, SBP systolic blood, DBP diastolic blood pressure, FBG fast blood glucose, $T G$ triglyceride, $T C$ total cholesterol, $H D L-C$ high- density lipoprotein cholesterol, LDL-C low-density lipoprotein cholesterol, eGFR estimated glome-rular filtration rate, CHD coronary heart disease, PWV pulse-wave velocity 
Table 2 Multiple linear regression analysis of baseline parameters and follow-up arterial stiffness

\begin{tabular}{|c|c|c|c|c|c|}
\hline & \multicolumn{2}{|c|}{ carotid-femoral PWV } & \multicolumn{3}{|c|}{ carotid-femoral PWV } \\
\hline & $r$ & $P$-value & $\beta$ & $\mathrm{Cl}$ & $P$-value \\
\hline \multicolumn{6}{|c|}{ All subjects $(n=1447)$} \\
\hline Age & 0.538 & $<0.001$ & -0.025 & $0.012 \sim 0.037$ & $<0.001$ \\
\hline Male & - & - & 0.725 & $0.340 \sim 1.110$ & $<0.001$ \\
\hline Smoking & - & - & 0.320 & $-0.086 \sim 0.725$ & 0.122 \\
\hline Diabetes & - & - & 0.232 & $0.058 \sim 0.522$ & 0.117 \\
\hline Hypertension & - & - & 0.883 & $0.501 \sim 1.265$ & $<0.001$ \\
\hline Hepcidin & 0.151 & $<0.001$ & 1.498 & $0.696 \sim 2.300$ & $<0.001$ \\
\hline $\mathrm{TG}^{\mathrm{a}}$ & 0.747 & $<0.001$ & 0.367 & $0.140 \sim 0.593$ & 0.002 \\
\hline $\mathrm{HDL}-\mathrm{C}^{\mathrm{a}}$ & 0.065 & 0.049 & 0.192 & $-0.382 \sim 0.767$ & 0.511 \\
\hline LDL-C & 0.014 & 0.679 & 0.741 & $0.240 \sim 1.242$ & $<0.004$ \\
\hline TC & 0.049 & 0.132 & 0.549 & $0.153 \sim 0.945$ & 0.007 \\
\hline SBP & 0.228 & $<0.001$ & 0.046 & $0.033 \sim 0.060$ & $<0.001$ \\
\hline DBP & -0.038 & 0.252 & 0.059 & $-0.079 \sim-0.040$ & $<0.001$ \\
\hline BMI & 0.054 & 0.098 & 0.090 & $0.037 \sim 0.144$ & $<0.001$ \\
\hline FBG & 0.118 & $<0.001$ & 0.293 & $0.187 \sim 0.398$ & $<0.001$ \\
\hline eGFR ${ }^{a}$ & -0.376 & 0.001 & -0.059 & $-0.072 \sim-0.047$ & $<0.001$ \\
\hline
\end{tabular}

TC total cholesterol, HDL-C high- density lipoprotein cholesterol, TG triglyceride, $L D L-C$ low-density lipoprotein cholesterol, SBP systolic blood pressure, DBP diastolic blood pressure, $B M I$ body mass index, FBG fast blood glucose, eGFR estimated glomerular filtration rate, PWV pulse wave velocity

a: natural logarithm transformed

§: Covariates in the multiple-adjusted models included age, gender, hypertension, DM, current smoking, levels of plasma TC, TG, LDL-C, HDL-C, SBP, DBP,FBG, BMI and eGFR

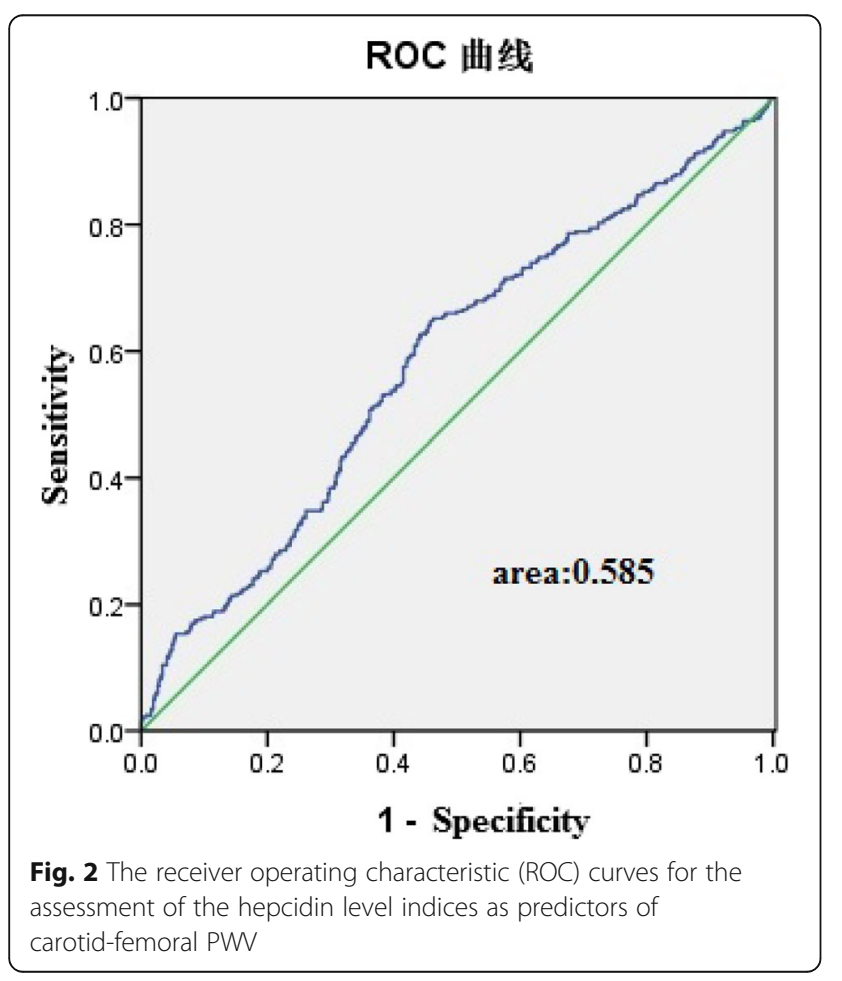

\section{Association of hepcidin with HDL subclasses (HDL2-C, HDL3-C)}

The associations between HDL subclasses as a continuous variable (natural logarithm transformed) and hepcidin at baseline are summarized in Table 3 . Hepcidin was significantly and negatively related to HDL3-C $(r=-0.197 ; P<0.001$, Fig. 3). In multivariable linear regression analysis, HDL3-C levels were negatively and independently associated with hepcidin at baseline $(\beta=-0.857, P=0.024)$. HDL2-C was not associated with hepcidin at baseline $(\beta=-1.121$, $P=0.133$ ).

ROC curves for the assessment of the association of hepcidin and HDL3-C is presented in Fig. 4.

\section{Discussion}

Until then, no studies had been conducted to prove the relationships among hepcidin levels, HDL3-C and carotid-femoral PWV. In the present longitudinal study, we demonstrated the following: 1) An association between baseline hepcidin and follow-up arterial stiffness independent of age, gender and other vascular risk factors. 2) An association between hepcidin and HDL3-C at baseline, which indicates that hepcidin may be a promising target to increase reverse cholesterol transport from macrophages and inhibit atherosclerosis. 
Table 3 Association of hepcidin with HDL subclasses (HDL2-C, HDL3-C)

\begin{tabular}{|c|c|c|c|c|c|c|}
\hline & \multicolumn{3}{|l|}{ HDL2-C } & \multicolumn{3}{|l|}{ HDL3-C } \\
\hline & $\bar{\beta}$ & $\mathrm{Cl}$ & $P$-value & $\bar{\beta}$ & $\mathrm{Cl}$ & $P$-value \\
\hline \multicolumn{7}{|c|}{ All subjects $(n=1447)$} \\
\hline hepc & -1.121 & $0.133 \sim-2.584$ & 0.133 & -0.857 & $-1.599 \sim-0.114$ & 0.024 \\
\hline Male & -4.243 & $-5.352 \sim-3.131$ & $<0.001$ & -0.602 & $-1.165 \sim-0.039$ & 0.036 \\
\hline age & 0.133 & $0.078 \sim 0.188$ & $<0.001$ & 0.033 & $0.005 \sim 0.061$ & 0.021 \\
\hline smoking & -0.001 & $0.171 \sim 1.076$ & 0.007 & -0.220 & $-0.835 \sim 0.395$ & 0.482 \\
\hline BMl & -0.098 & $-0.188 \sim-0.008$ & 0.032 & 0.001 & $-0.047 \sim 0.045$ & 0.960 \\
\hline TC & 9.042 & 7.993 10.090 & $<0.001$ & 2.894 & $2.362 \sim 3.426$ & $<0.001$ \\
\hline LDL-C & -0.892 & $-10.097 \sim-7.358$ & 0.002 & -1.450 & $-2.146 \sim-0.755$ & $<0.001$ \\
\hline $\mathrm{TG}^{\mathrm{a}}$ & -9.835 & $-10.840 \sim-8.810$ & $<0.001$ & -1.688 & $-2.203 \sim-1.172$ & $<0.001$ \\
\hline FBG & -0.265 & $-0.580 \sim 0.050$ & 0.099 & 0.044 & $-0.116 \sim 0.204$ & 0.589 \\
\hline eGFR ${ }^{a}$ & 0.060 & $0.016 \sim 0.104$ & 0.008 & 0.013 & $-0.010 \sim 0.035$ & 0.262 \\
\hline
\end{tabular}

TC total cholesterol, HDL-C high- density lipoprotein cholesterol, TG triglyceride, $L D L-C$ low-density lipoprotein cholesterol, BMI body mass index, FBG fast blood glucose, eGFR estimated glomerular filtration rate, PWV pulse wave velocity

a: natural logarithm transformed

§: Covariates in the multiple-adjusted models included age, gender, hypertension, DM, current smoking, levels of plasma TC,TG,HDL-C,LDL-C,FBG, BMI, eGFR

Sullivan proposed that hepcidin would increase the risk of atherosclerosis by increasing iron aggregation in macrophages, and subsequently increase lipid peroxidation and foam cell formation within atherosclerotic plaques [18]. A study that included 766 participants aged 46 to 67 years confirmed that hepcidin affected the development of atherosclerosis in women [19]. Valenti L et al. that increased hepcidin levels had positive association with increased aortic stiffness independently in 827 consecutive outpatients [20]. Furthermore, it is founded that hepcidin-25 was independently associated with carotid plaques in patients with nonalcoholic fatty liver disease [21].

In our study, we used carotid-femoral PWV, which reflects the presence of atherosclerosis. Recently, the risk factor that arterial stiffness play in subclinical vascular disease as well as cardiovascular mortality has been recognized [22-25]. Several studies based on community population have attached importance to the role of arterial stiffness,

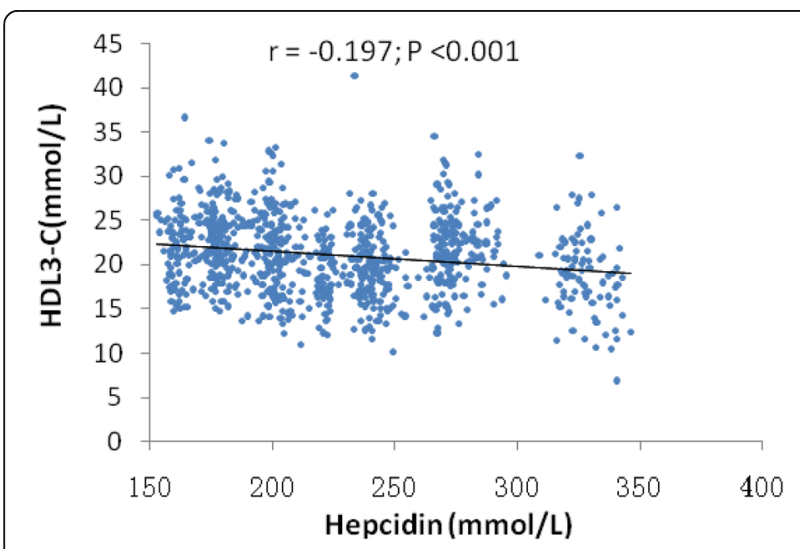

Fig. 3 Hepcidin was significantly and negatively related to HDL3-C which can be measured by the noninvasive technique pulse wave velocity (PWV) [26, 27]. Carotid-femoral PWV is the "gold standard" for the assessment of arterial stiffness; numerous epidemiological studies indicate its predictive value for CHD [28]. In addition, our research population is older, nearly half of them are over 65 . The steepest rise of transmural pressure-induced arterial wall damage occurs after the age of 60 years [29-31], increasing the risk of ill effects due to other risk factors in older subjects.

In the past, the research focused on hepcidin and abnormal iron metabolism and CV risk; our current research identified an association between hepcidin and HDL3-C at baseline, which indicates that the HDL3-C

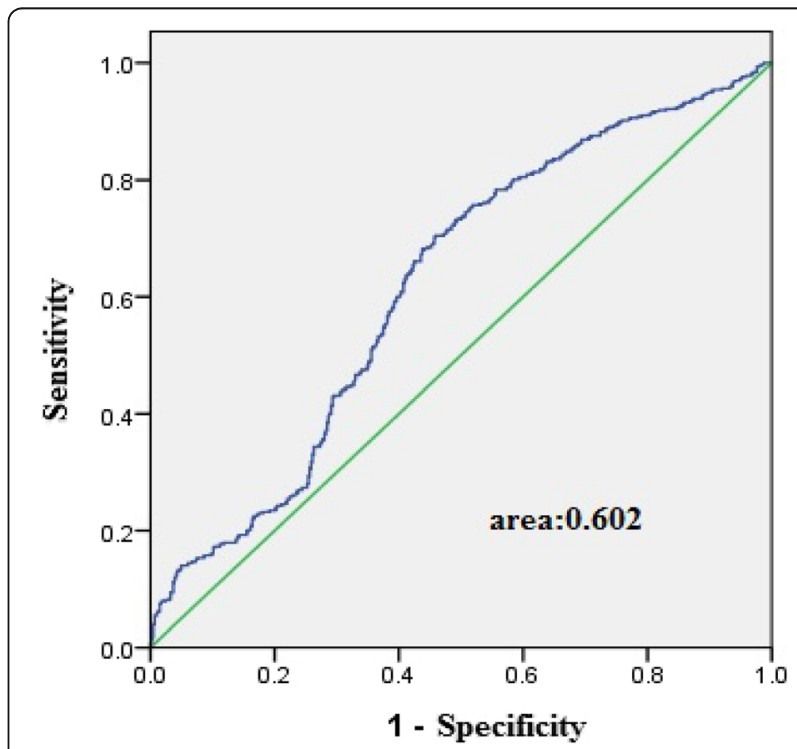

Fig. 4 The receiver operating characteristic (ROC) curves for the assessment of the association of hepcidin and HDL3-C 
level may reflect the change in cholesterol efflux from peripheral arteries and partly explain the relationship between hepcidin and the change of arterial stiffness. Some potential mechanisms support this hypothesis that hepcidin is a biomarker of carotid-femoral PWV risk, given the role of decreasing cholesterol efflux that has been observed in vitro or in vivo. In an experimental mouse study [32], the suppression of hepcidin production in the liver decreases the formation of foam cells and atherosclerosis by reducing intracellular iron content in macrophages, which increased the efflux cholesterol capacity. Hepcidin has been showed to increase reactive oxygen species and decrease cholesterol efflux by increasing intracellular iron content, which was done with human monocytes derived from atherosclerotic plaques [33]. These findings are identical to other in vivo and in vitro studies [34], showing that the interaction of (locally produced) accumulated lipids with hepcidin and trapped iron play a key role in the proatherosclerotic activation of macrophages and accelerates plaque destabilization. Moreover, Omar Saeed et al. reported that the pharmacologic suppression of hepcidin increases the expression of ABCA1 and ABCG1 and lipid efflux via the macrophage-specific expression of cholesterol efflux transporters and reduces foam cell formation and atherosclerosis [12]. Clinically, we found that the hepcidin level was negatively associated with the HDL3-C level among subjects at baseline, indicating that hepcidin may decrease cholesterol efflux, manifested as lower HDL3-C. HDL-C can been isolated into HDL2-C ('large, buoyant') and HDL3-C ('small, dense') particles, respectively, by ultracentrifugation, and HDL particles are heterogeneous in their structure, metabolism, and biological functionality [35-37]. It has been demonstrated that protein-rich HDL3 particles are even more important to atheroprotective than cholesterol-rich HDL2 particles [38-40]. HDL 3 is the main component of HDL; the majority $(75 \%)$ of HDL cholesterol resides in this subclass. Martin SS et al. reported that a low HDL3-C level, but not a low HDL2-C level, was associated with an increased risk of myocardial infarction or death in secondary prevention patients, highlighting the potential value of HDL3-C [41]. HDL3 particles play a central role in RCT, extracting cholesterol from the periphery, and maturing into HDL2 particles via progressive lipidation by lecithin: cholesteryl acyltransferase [41]. The central positioning of HDL3 in RCT and greater contribution to total HDL-C may indicate that HDL3 assumes the majority of the responsibility for HDL functions, such as RCT.

The above data proves this conclusion that the HDL3-C level may reflect the change in cholesterol efflux from peripheral arteries and partly explains the relationship of hepcidin and change in arterial stiffness, but further research is needed to explore more of these mechanisms.

\section{Conclusions}

We found an association between baseline hepcidin and follow-up arterial stiffness that was independent of age, gender and other vascular risk factors. We also identified an association between hepcidin and HDL3-C at baseline, which indicates that the HDL3-C level may reflect the change in cholesterol efflux from peripheral arteries and partly explain the relationship between hepcidin and the change of arterial stiffness.

\section{Abbreviations \\ BMI: Body mass index; cf-pwv: Arotid-femoral pulse wave velocity; cr- pwv: Carotid-radial pulse wave velocity; DBP: Diastolic blood pressure; DM: Diabetes mellitus; eGFR: Estimated glomerular filtration rate; FBG: Fasting blood glucose; HDL-C: High-density lipoprotein cholesterol; LDL-C: Low- density lipoprotein cholesterol; SBP: Systolic blood; TC: Total cholesterol; TGs: Triglycerides}

\section{Acknowledgements}

Acknowledgments and disclosures: We thank colleagues at the Department of Laboratory Medicine, the PLA General Hospital for help with biochemical measurements. We are also grateful to all study participants for their participation in the study.

\section{Funding}

This research is supported by a grant from the Key National Basic Research Program of China (2013CB530804) and the Key Science and Technology Foundation of China (2012ZX09303004-002) to Dr. Ping Ye.

\section{Availability of data and materials \\ All data generated or analysed during this study are included in this published article.}

\section{Authors' contributions}

$X W, S L$ and PY designed the study; RC, XY, WX, YZ and HW participated in acquisition of data; XW, PY and YB researched and evaluated the literature; XW undertook the statistical analysis and wrote the first draft of the manuscript. All authors read and approved the final manuscript.

\section{Ethics approval and consent to participate}

The study was approved by the ethics committee of the People's Liberation Army General Hospital, and each subject provided informed written consent.

Consent for publication

Not applicable.

\section{Competing interests}

The authors declare that they have no competing interests.

\section{Publisher's note}

Springer Nature remains neutral with regard to jurisdictional claims in published maps and institutional affiliations.

Received: 3 July 2018 Accepted: 11 September 2018 Published online: 30 October 2018

\section{References}

1. Sullivan JL. Iron and the sex differences in heart disease risk. Lancet. 1981;1:1293-4.

2. Kiechl S, Willeit J. The natural course of atherosclerosis part I: incidence and progression. Arterioscler Thromb Vasc Biol. 1999;19:1484-90.

3. Salonen JT, Nyyssönen $\mathrm{K}$, Korpela $\mathrm{H}$, et al. High stored iron levels are associated with excess risk of myocardial infarction in eastern Finnish men. Circulation. 1992;86:803-11.

4. Kiechl S, Willeit J, Egger G, et al. Body iron stores and the risk of carotid atherosclerosis: prospective results from the broneck study. Circulation. 1997;96:3300-7.

5. Park $\mathrm{CH}$, Valore $\mathrm{EV}$, Waring $\mathrm{AJ}$, et al. Hepcidin, a urinary antimicrobial peptide synthesized in the liver. J Biol Chem. 2001;276:7806-10. 
6. Pigeon C, llyin G, Courselaud B, et al. A new mouse liver-specific gene, encoding a protein homologous to human antimicrobial peptide hepcidin, is overexpressed during iron overload. J Biol Chem. 2001;276:7811-9.

7. Nemeth E, Ganz T. Regulation of iron metabolism by hepcidin. Annu Rev Nutr. 2006;26:323-42.

8. Ganz T. Hepcidin - a regulator of intestinal iron absorption and iron recycling by macrophages. Best Pract Res Clin Haematol. 2005;18:171-82.

9. Kuragano T, Itoh K, Shimonaka Y, et al. Hepcidin as well as TNF-alpha are significant predictors of arterial stiffness in patients on maintenance hemodialysis. Nephrol Dial Transplant. 2011;26:2663-7.

10. Valenti L, Dongiovanni P, Motta BM, et al. Serum hepcidin and macrophage iron correlate with MCP-1 release and vascular damage in patients with metabolic syndrome alterations. Arterioscler Thromb Vasc Biol. 2011;31:683-90.

11. Valenti $L$, Swinkels DW, Burdick $L$, et al. Serum ferritin levels are associated with vascular damage in patients with nonalcoholic fatty liver disease. Nutr Metab Cardiovasc Dis. 2011;21:568-75.

12. Saeed O, Otsuka F, Polavarapu R, et al. Pharmacological suppression of hepcidin increases macrophage cholesterol efflux and reduces foam cellformation and atherosclerosis. Arterioscler Thromb Vasc Biol 2012;32(2):299-307.

13. Kontush A, Chapman MJ. Antiatherogenic small, dense HDL-guardian angel of the arterial wall? Nat Clin Pract Cardiovasc Med. 2006:3:144-53.

14. Laurent S, Cockcroft J, Van Bortel L, et al. Expert consensus document on arterial stiffness: methodological issues and clinical applications. Eur Heart J. 2006;27:2588-605.

15. Hirano T, Nohtomi K, Koba S, et al. A simple and precise method for measuring HDL-cholesterol subfractions by a single precipitation followed by homogenous HDL-cholesterol assay. J Lipid Res. 2008;49:1130-6.

16. McEniery C, Cockcroft JR. Does arterial stiffness predict atherosclerotic coronary events? Adv Cardiol. 2007:44:160-72.

17. Blacher J, Guerin AP, Pannier B, et al. Impact of aortic stiffness on survival in end-stage renal disease. Circulation. 1999;99:2434-9.

18. Sullivan JL. Macrophage iron, hepcidin, and atherosclerotic plaque stability. Exp Biol Med (Maywood). 2007;232:1014-20.

19. Galesloot TE, Holewijn S, Kiemeney LA, et al. Serum hepcidin is associated with presence of plaque in postmenopausal women of a general population. Arterioscler Thromb Vasc Biol. 2014;34(2):446-56.

20. Valenti L, Maloberti A, Signorini S, et al. Iron stores, Hepcidin, and aortic stiffness in individuals with hypertension. PLoS One. 2015;10(8):e0134635.

21. Valenti L, Swinkels DW, Burdick $L$, et al. Serum ferritin levels are associated with vascular damage in patients with nonalcoholic fatty liver disease. Nutr Metab Cardiovasc Dis. 2011;21:568-75.

22. van Popele NM, Grobbee DE, Bots ML, et al. Association between arterial stiffness and atherosclerosis: the Rotterdam study. Stroke. 2001;32:454-60.

23. Zureik M, Temmar M, Adamopoulos C, et al. Carotid plaques, but not common carotid intimamedia thickness, are independently associated with aortic stiffness. J Hypertens. 2002;20:85-93.

24. Blacher J, Guerin AP, Pannier B, et al. Impact of aortic stiffness on survival in end-stage renal disease. Circulation. 1999;99:2434-9.

25. Laurent $\mathrm{S}$, Boutouyrie $\mathrm{P}$, Asmar $\mathrm{R}$, et al. Aortic stiffness is an independent predictor of all-cause and cardiovascular mortality in hypertensive patients. Hypertension. 2001;37:1236-41.

26. Avolio AP, Chen S, Wang R, et al. Effects of aging on changing arterial compliance and left ventricular load in a northern Chinese urban community. Circulation. 1983;68:50-8.

27. Asmar R, Benetos A, Topouchian J, et al. Assessment of arterial distensibility by automatic pulse wave velocity measurement: validation and clinical application study. Hypertension. 1995;26:485-90.

28. Laurent S, Cockcroft J, Van Bortel L, et al. Expert consensus document on arterial stiffness: methodological issues and clinical applications. Eur Heart J. 2006;27:2588-605.

29. Franklin SS, Gustin W 4th, Wong ND, et al. Hemodynamic patterns of agerelated changes in blood pressure. The Framingham heart study. Circulation. 1997:96:308-15.

30. O'Rourke MF. Arterial function in health and disease. Edinburgh, UK: Churchill-Livingstone; 1982.

31. Folkow B. Structure and function of the arteries in hypertension. Am Heart J. 1987;114:938-48.

32. Saeed O, Otsuka F, Polavarapu R, et al. Pharmacological suppression of hepcidin increases macrophage cholesterol efflux and reduces foam cell formation and atherosclerosis. Arterioscler Thromb Vasc Biol. 2012;32:299-307.
33. Finn AV, Nakano M, Polavarapu R, et al. Hemoglobin directs macrophage differentiation and prevents foam cell formation in human atherosclerotic plaques. J Am Coll Cardiol. 2012;59:166-77.

34. Li JJ, Meng X, Si HP, et al. Hepcidin destabilizes atherosclerotic plaque via overactivating macrophalages after erythrophagocytosis. Arterioscler Thromb Vasc Biol. 2012;32:1158-66.

35. Assmann G, Gotto AM. HDL cholesterol and protective factors in atherosclerosis. Circulation. 2004;109:|I18-III14.

36. Davidson WS, Silva RA, Chantepie S, Lagor WR, Chapman MJ, Kontush A. Proteomic analysis of defined HDL subpopulations reveals particle-specific protein clusters: relevance to antioxidative function. Arterioscler Thromb Vasc Biol. 2009;29:870-6.

37. Rosenson RS, Brewer HB Jr, Davidson WS, et al. Cholesterol efflux and atheroprotection: advancing the concept of reverse cholesterol transport. Circulation. 2012:125:1905-19.

38. Du XM, Kim MJ, Hou L, et al. HDL particle size is a critical determinant of ABCA1-mediated macrophage cellular cholesterol export. Circ Res. 2015; 116(7):1133-1142.

39. Camont L, Chapman MJ, Kontush A. Biological activities of HDL subpopulations and their relevance to cardiovascular disease. Trends Mol Med. 2011;17(10):594-603.

40. Rached FH, Chapman MJ, Kontush A. HDL particle subpopulations: focus on biological function. Biofactors. 2015;41(2):67-77.

41. Martin SS, Khokhar AA, May HT, et al. HDL cholesterol subclasses, myocardial infarction, and mortality in secondary prevention: the lipoprotein investigators collaborative. Eur Heart J. 2015:36(1):22-30.

\section{Ready to submit your research? Choose BMC and benefit from:}

- fast, convenient online submission

- thorough peer review by experienced researchers in your field

- rapid publication on acceptance

- support for research data, including large and complex data types

- gold Open Access which fosters wider collaboration and increased citations

- maximum visibility for your research: over $100 \mathrm{M}$ website views per year

At BMC, research is always in progress.

Learn more biomedcentral.com/submissions 